\section{Age, sex and period estimates of Australia's mental health over the last I 7 years}

Australian \& New Zealand Journal of Psychiatry I-7 DOI: $10.1177 / 0004867419888289$

(C) The Royal Australian and New Zealand College of Psychiatrists 2019 Article reuse guidelines: sagepub.com/journals-permissions journals.sagepub.com/home/anp @SAGE

\author{
Richard A Burns ${ }^{1}$ D, Peter Butterworth ${ }^{1,2}$ and \\ Dimity A Crisp ${ }^{3}$ D
}

\begin{abstract}
Objective: Describing the long-term mental health of Australians is limited as many reports rely on cross-sectional studies which fail to account for within-person changes and age-related developmental processes which may bias estimates which ignore these phenomena. We examined the 17-year trajectories of mental health in 27,519 Australian adults.
\end{abstract}

Methods: Household panel data of 27,519 participants aged I8years and over from the Household, Income and Labour Dynamics in Australia Survey provided at least one observation of mental health over a 17-year period from 200I. On average, participants reported 7.6 observations. Mental Health was assessed annually using the Short-Form Health Survey-36 mental health scale.

Results: Over time, there were only very small changes in mental health and only for the youngest and oldest adults. Over time, there was consistent evidence for better metal health with increasing age, although for the very old, there appear to be substantial declines. These patterns were consistent between sex. In line with an existing literature, males reported better mental health over life span, although the declines of mental health in very-late-life are particularly pronounced for males.

Conclusion: Decline in mental health was only reported by the youngest and oldest respondents, and was notable only in the last 4-5 years. However, the magnitude of the decline was small and further follow-up will be needed to determine whether this is a trend of substantive declining mental health for these specific age cohorts. In contrast, the more consistent finding is that there has been no substantive change in the level of mental health in Australia over the last 17 years. Analysis of the mental health trajectories of baseline age-cohorts confirmed that age differences are consistent over time.

\title{
Keywords
}

Mental health, trajectories, age differences, sex differences, Australian population

The burden of mental illness is clear and substantive. Recent estimates suggest the global burden of mental illness accounts for $32.4 \%$ of years lived with disability (YLDs) and $13.0 \%$ of disability-adjusted life-years (DALYs) (Vigo et al., 2016). These estimates are almost double those previously suggested (Whiteford et al., 2013) and place mental illness as the most significant driver of YLDs, and comparable with cardiovascular and circulatory diseases in terms of the impact on DALYs (Vigo et al., 2016). Precise estimates of mental health in Australia are predominantly drawn from cross-sectional panel designs, including the 1997 and 2007 Australian National Survey of Mental Health and Wellbeing (NSMHWB) (Slade et al., 2009) and multiple national health surveys (ABS, 2002, 2015). In line with international patterns, sex and age differences are frequently reported; younger adults and younger females typically report poorer mental health outcomes (Slade et al., 2009). A recent

'Centre for Research on Ageing, Health \& Wellbeing, Research School of Population Health, The Australian National University, Canberra, ACT, Australia

2Melbourne Institute of Applied Economic and Social Research, Faculty of Business and Economics, The University of Melbourne, Melbourne, VIC, Australia

${ }^{3}$ Centre for Applied Psychology, Faculty of Health, University of Canberra, Canberra, ACT, Australia

Corresponding author:

Richard Andrew Burns, Centre for Research on Ageing, Health \& Wellbeing, Research School of Population Health, The Australian National University, Canberra, ACT 0200, Australia.

Email: Richard.Burns@anu.edu.au 
analysis of the National Health Surveys from 2001 to 2015 has identified no substantive change in Australians' mental health Jorm (2018) despite concerted public health initiatives and increased public funding. While these findings are drawn from large national surveys, and in the case of the NSMHWB include comprehensive batteries of mental health, the nature of their cross-sectional design precludes examining the experiences of mental health within the same individuals as they age. Given the role of developmental processes and age-related contextual factors that drive mental health outcomes, it is important to quantify the extent to which reported sex and age population estimates are consistent within individuals as they age, over a long follow-up period.

Large cross-sectional national surveys are valuable for assessing prevalence of mental health at a specific point in time, or for specific populations, but they fail to consider the impact of lifespan or developmental factors on mental health and psychological distress. Repeated cross-sectional designs preclude separation of cohort (e.g. the time of an individual's birth), period (the time the participant is observed) and ageing (developmental) trends. Longitudinal data are needed to disentangle these issues, and there is limited research available to allow for examination of birth, period and age differentials on mental health (Bell and Jones, 2015; Keyes et al., 2014). Longitudinal studies provide added efficiency to estimates that are otherwise drawn from repeated cross-sectional samples and which ignore within-person heterogeneity. In an Australian context, some longitudinal studies report mental health changes that are to a degree contradictory to the outcomes from large cross-sectional studies like the NSMHWB (Anstey et al., 2010; Burns et al., 2012, 2013a). For example, one very large study of mid and late-life adults identified that rates of psychological distress among older males in both NSMHWB surveys were much lower than those derived from large longitudinal studies of ageing which followed the same individuals over time (Burns et al., 2012, 2013b). This paper examines the trajectory of mental health in the Australian population over a 17-year period using longitudinal data from the nationally representative and longitudinal Household, Income and Labour Dynamics in Australia (HILDA) study and to quantify age and sex differentials in these trajectories.

\section{Method}

\section{Participants}

Participants were from the HILDA Survey (Wooden and Watson, 2007). HILDA is a longitudinal household panel survey that has been conducted annually since 2001. At baseline, there were 7682 sampled households resulting in interviews with 13,969 individual participants. Over time, new participants entered the study as they joined existing households. Results in this paper reflect responses of participants $(N=27,519)$ who provided at least one observation (mean number of responses $=7.6$ observations) on the relevant measures over the first 17 years of data provided by each household member aged 18 years and older via both personal interview and self-completion questionnaire. Overall, $5094(17.2 \%)$ participants provided one assessment, 6703 (23.0) provided between two and four observations, 7747 (26.0) provided between five and nine observations and $10,050(34.0 \%)$ provided 10 or more observations. 4073 participants (13.8\%) provided observations at all 17 waves. A flow of participants is described in Supplementary Table 1. Of the original baseline sample, 12,933 participants responded on the relevant mental health scale, and of whom, 4073 (31.4\% of the original sample) provided complete data at all waves. Using a household sampling frame, new entrants were introduced to the sample at each wave if they had moved into participating households. Notably, a top-up sample to ensure the representativeness of the HILDA study with the Australian population can be seen in Wave 11. At each wave, a number of participants who did not provide observations were either lost completely or simply did not participate at a particular wave, but returned at a later wave.

\section{Measures}

Mental Health. Mental Health was operationalised by the Mental Health Index subscale (MHI-5) of the Short-Form Health Survey-36 (SF-36) (Ware and Sherbourne, 1992) which has been validated in HILDA (Butterworth and Crosier, 2004). The MHI-5 has been used in epidemiological studies as an indicator of psychological distress (Gill et al., 2006; Rumpf et al., 2001; Skapinakis et al., 2005; Yamazaki et al., 2005) and comprises five items including, 'Been a nervous person', 'Felt so down in the dumps nothing could cheer you up', 'Felt calm and Peaceful', 'Felt down' and 'Been a happy person'. The MHI-5 has excellent sensitivity and specificity for mood disorders in particular with a receiver operating curve (AUC) of 0.88, and an AUC of 0.72 , for identifying any DSM-IV Axis 1 disorders excluding substance use disorder (Rumpf et al., 2001). MHI-5 scores range from 0 (high level of disability) to 100 (low level of disability). A number of cut-offs have been proposed as a basis for screening for possible Axis 1 disorders, but the MHI-5 appears to have particular utility in detecting mood disorder. For mood disorders, a cut-off of 60 points or less reported a sensitivity of 0.83 and specificity of 0.78 . In contrast, anxiety reported sensitivity of 0.52 and specificity of 0.78 , and for any Axis 1 disorder, sensitivity was 0.60 and specificity was 80 (Rumpf et al., 2001).

\section{Statistical analysis}

All analyses were undertaken and graphs created in STATA SE v.15 (StataCorp, 2015). Analyses were undertaken 
Table I. Tests of age, sex and time associations with mental health over the 17 years of the HILDA study.

\begin{tabular}{|lccc|} 
& \multicolumn{3}{c}{ Mental Health } \\
\cline { 2 - 4 } & \multicolumn{1}{c}{$\chi^{2}$} & $d f$ & $p$ \\
\hline Age & 253.59 & 6 & $<0.001$ \\
\hline Sex & 150.69 & 1 & $<0.001$ \\
\hline Time & 230.07 & 16 & $<0.001$ \\
\hline Age $\times$ Sex & 29.97 & 6 & $<0.001$ \\
\hline Age $\times$ Time & 284.99 & 96 & $<0.001$ \\
\hline Male $\times$ Time & 10.39 & 16 & 0.846 \\
\hline Age $\times$ Sex $\times$ Time & 75.94 & 96 & 0.935 \\
\hline
\end{tabular}

HILDA: Household Income and Labour Dynamics in Australia.

within a multi-level linear regression framework. These models provide maximum likelihood estimates and standard errors which are adjusted for the clustering of repeated observations within individuals who were followed over time. Age, sex and time point estimates were derived from a full-factorial model that included all main effects for age, sex and time, and all two- and three-way interactions between these factors. Point estimates were then saved using a post-estimation margins command, which were then plotted.

In line with recommend practice (Wasserstein et al., 2019), we report exact significance values and emphasise differences and changes in mental health in terms of the magnitude of the effect sizes reported. The magnitude of the effect sizes can be interpreted in two ways. First, a comparison can be made in regard to the recommended cut-off of 60 for mood disorder. Second, we can estimate the magnitude of changes in mental health score in terms of standardised effect sizes. Within this sample, small $(d=0.20)$, medium $(d=0.40)$ and large $(d=0.60)$ effect sizes corresponded with changes in the mental health scale of 2 points for small effect sizes, 4 points for medium effect sizes and 8 points for large effect sizes. These effect sizes reflect changes in standard deviation terms; hence, a difference of 2 points on the mental health scale reflects a magnitude difference of 0.2 standard deviations. Similarly, a difference of 8 points on the mental health scale reflects a magnitude difference of 0.6 standard deviations.

\section{Results}

Age, sex and temporal estimates of mental health were obtained from a full-factorial model that included all main, two- and three-way interaction terms (Table 1). Over the 17 years, the mean level of mental health was $\mathrm{M}=72.96$ $(\mathrm{SE}=0.09)$. There was a significant difference between sex $(z=13.46 ; \quad p<0.001) ; \quad$ males $\quad(\mathrm{M}=74.20 ; \quad \mathrm{SE}=0.13)$ reported comparatively higher (better) levels of mental health than females $(\mathrm{M}=71.81 ; \mathrm{SE}=0.12)$. While there were significant two-way interactions between age and sex, and age and time, there were no differences reported in the three-way Age $\times$ Sex $\times$ Time interaction, $\chi^{2}(96)=75.94$; $p=0.935$, and two-way Sex $\times$ Time interaction, $\chi^{2}(16)=10.39 ; p=0.846$.

Estimates of mental health by age group averaged over the study period are reported in Supplementary Table 2. Estimates are provided for the whole sample and by sex. Sex $\times$ Age trajectories can be visually compared in Figure 1 for both sexes. Overall, there appears to be a general trend of improvement in mental health, $\chi^{2}(6)=253.59, p<0.001$, with increasing age. That is, the poorest levels of mental health were reported by younger adults. This pattern was consistent for males and females, although females reported greater improvement in mental health with age, $\chi^{2}(6)=$ $29.97, p<0.001$. Notably, there was a small decline in level of mental health among those males aged $75+$ in contrast to those aged $65-74$ years.

There were declines in level of mental health, $\chi^{2}(16)=230.07, p<0.001$, over the 17 years of the HILDA study, and this was consistent for males and females, $\chi^{2}$ $(16)=10.39, p=0.846$. Estimates for each year of the study are reported in Supplementary Table 3 by sex and in Supplementary Table 4 by age groups. However, the size of change in mental health was only of a small magnitude from $\mathrm{M}=73.55(\mathrm{SE}=0.14)$ in 2001 to $\mathrm{M}=72.30(\mathrm{SE}=0.13)$ in 2017 and appears to occur in the last 5 years only. These differences were less than 2 points on the mental health scale and reflect a change far less than 0.2SD that would reflect a small effect size.

Examination of the separate sex and age estimates over time suggests that these changes are not consistent for all age groups. That is, any overall pattern of change was driven only by certain age groups. Those aged $18-24$ years reported a non-linear change with an increase from $71.36(\mathrm{SE}=0.36)$ at baseline to $73.16(\mathrm{SE}=0.41) 8$ years later, followed by a decline to $69.62(\mathrm{SE}=0.35)$ at the final observation in 2017. In contrast, those aged 25-34 years appear to report stability for the first 13 years from baseline $(\mathrm{M}=72.54 ; \mathrm{SE}=0.27)$ before gradually declining in the last few years to 2017 $(\mathrm{M}=70.34 ; \mathrm{SE}=0.27)$. Perhaps, most notable was the decline in mental health of the oldest participants, declining from $76.97(\mathrm{SE}=0.53)$ at baseline to $73.33(\mathrm{SE}=0.38)$ at the final observation. Less substantive changes were reported by those aged 35-44, 45-54 and 65-74years with only gradual declines in the final waves of observation while those aged 55-64years reported a small increase. Indeed, plotting the trajectories of mental health by sex (Figure 2) and by age group (Figure 3 ) more clearly reveals that the declines in level of mental health are really only notable in the last 4 years, and this pattern is only evident among younger (18-34 year olds) and older (75+) adults. Further 
Table 2. Average 17-year estimate of change in level of mental health for the whole sample, and separately by sex and age groups.

\begin{tabular}{|c|c|c|}
\hline & \multicolumn{2}{|l|}{ Mental Health } \\
\hline & $\beta(\mathrm{SE})$ & $p$ \\
\hline \multicolumn{3}{|l|}{ Whole sample } \\
\hline Intercept & $73.10(0.10)$ & $<0.001$ \\
\hline I7-year estimate of change & $-0.26(0.09)$ & 0.004 \\
\hline \multicolumn{3}{|l|}{ Male } \\
\hline Intercept & $74.48(0.15)$ & $<0.001$ \\
\hline I7-year estimate of change & $-0.53(0.13)$ & $<0.001$ \\
\hline \multicolumn{3}{|l|}{ Female } \\
\hline Intercept & $71.84(0.14)$ & $<0.001$ \\
\hline 17-year estimate of change & $-0.05(0.13)$ & 0.738 \\
\hline \multicolumn{3}{|l|}{ 18-24 age group } \\
\hline Intercept & $72.54(0.31)$ & $<0.001$ \\
\hline I7-year estimate of change & $-2.12(0.46)$ & $<0.001$ \\
\hline \multicolumn{3}{|l|}{ 25-34 age group } \\
\hline Intercept & $73.23(0.24)$ & $<0.001$ \\
\hline 17-year estimate of change & $-1.59(0.33)$ & $<0.001$ \\
\hline \multicolumn{3}{|l|}{$35-44$ age group } \\
\hline Intercept & $73.09(0.22)$ & $<0.001$ \\
\hline 17-year estimate of change & $-0.86(0.31)$ & 0.006 \\
\hline \multicolumn{3}{|l|}{ 45-54 age group } \\
\hline Intercept & $73.4 I(0.24)$ & $<0.001$ \\
\hline I7-year estimate of change & $-0.36(0.32)$ & 0.258 \\
\hline \multicolumn{3}{|l|}{ 55-64 age group } \\
\hline Intercept & $74.07(0.28)$ & $<0.001$ \\
\hline 17-year estimate of change & $1.28(0.34)$ & $<0.001$ \\
\hline \multicolumn{3}{|l|}{ 65-74 age group } \\
\hline Intercept & $77.27(0.31)$ & $<0.001$ \\
\hline 17-year estimate of change & $-0.91(0.38)$ & 0.017 \\
\hline \multicolumn{3}{|l|}{$75+$ age group } \\
\hline Intercept & $77.93(0.37)$ & $<0.001$ \\
\hline I7-year estimate of change & $-4.43(0.4 I)$ & $<0.001$ \\
\hline
\end{tabular}

SE: standard error.

follow-up of the HILDA sample will be needed to determine whether this reflects a continuing trend of worsening mental health in Australia. In contrast, for most other age groups, the changes have either not been substantial or do not necessarily reflect worsening mental health.

The average 17-year change estimates are provided in Table 2 to quantify the magnitude of these changes. Overall
Figure I. Point estimates and corresponding $95 \%$

confidence intervals of Mental Health scores by age and sex groups averaged over the 17 years of HILDA.

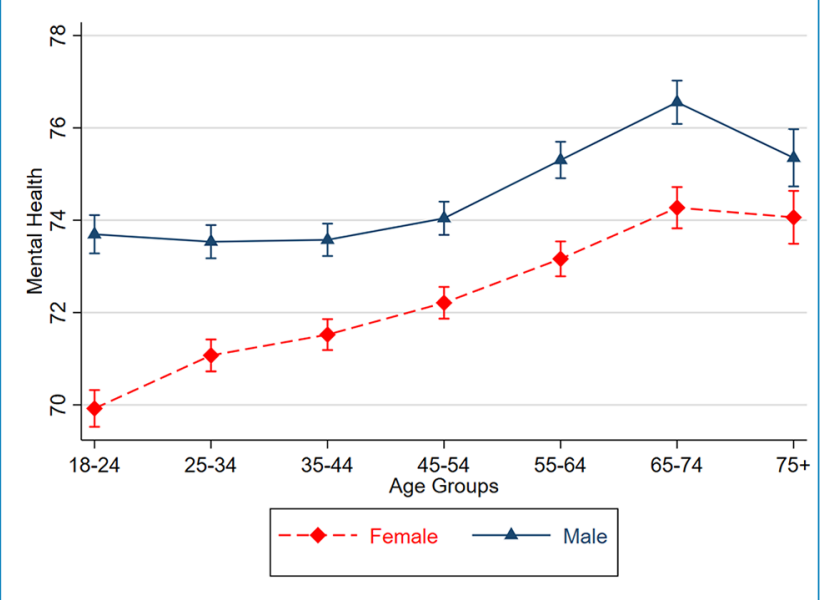

Figure 2. Point estimates and corresponding $95 \%$

confidence intervals in mental health trajectories over the 17 years of HILDA, by sex.

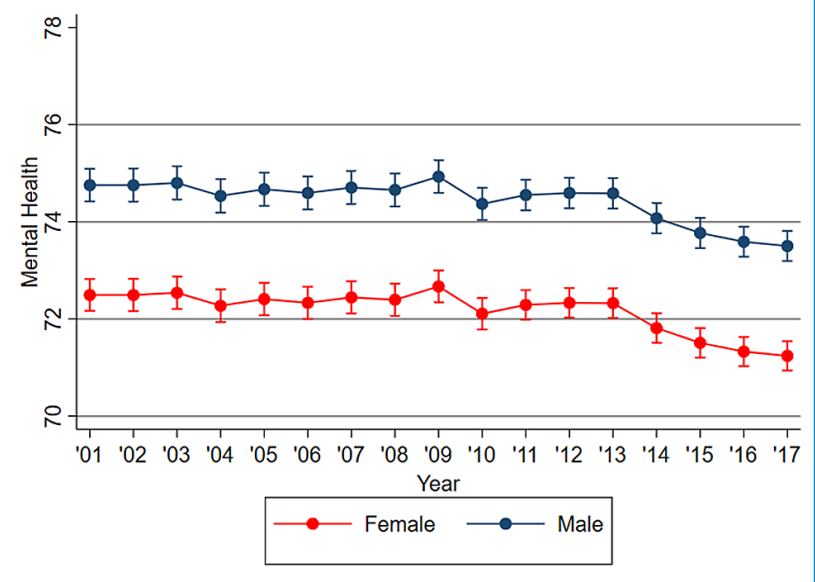

there was only a very small decline in mental health over the 17 years $(\beta=-0.26$; $\mathrm{SE}=0.09)$. Given the scale of the SF-36 scale, this is a change of only very small magnitude particularly since most age-sex estimates over the 17 years of HILDA are in the low to mid-70s range, far from the recommended cut-off of 60 for mood disorder. There was no overall change in level of mental health for females, $\beta=-0.05(\mathrm{SE}=0.13)$, but there was a decline in level of mental health for males, $\beta=-0.53(\mathrm{SE}=0.13)$. There was substantial variation in the amount of change mental health by age group. For example, the 18-24 and 25-34 age groups, who already reported the lowest levels of mental health, reported substantial declines of between $\beta=-2.12$ $(\mathrm{SE}=0.45)$ and $\beta=-1.59(\mathrm{SE}=0.33)$. These correspond to small effect sizes of $d \leqslant 0.20$. However, most notable was 
Figure 3. Point estimates and corresponding $95 \%$ confidence intervals in mental health trajectories over the I7years of HILDA, by age group.

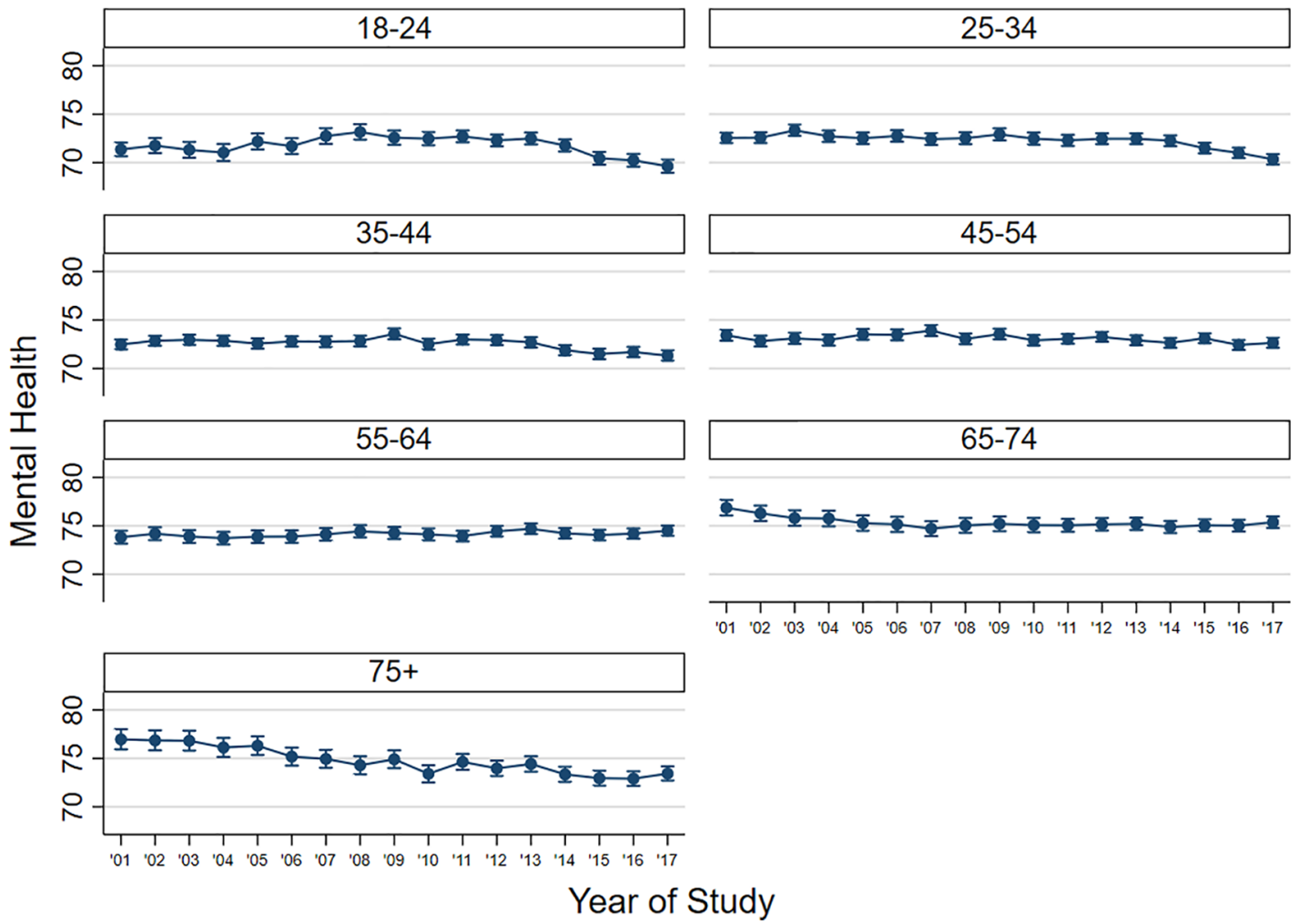

the more substantive decline of $\beta=-4.44(\mathrm{SE}=0.41)$ for those aged $75+$, corresponding with a moderate effect size. In contrast, the 45-54 age group reported no change over the 17 years, while the 55-64 age group reported small increases of $\beta=1.30(\mathrm{SE}=0.34)$.

\section{Discriminating age-related differences from birth cohort effects}

As a form of sensitivity analysis, and in order to confirm that age and sex differences over the last 17 years were not related to birth cohort effects, we estimated the trajectories of mental health scores among individuals according to their baseline age. In this way, we could estimate mental health trajectories as individuals aged through the HILDA study. For example, over the 17-year study period, an 18 year old at baseline would be 35 in the final observation used in this paper. Figure 4 presents these baseline agegroup mental health trajectory estimates for males and females as reflected by the separate trajectories in grey. As a comparison, the mean estimate for mental health for those who were not at baseline is also provided and reflected by single trajectory in black. Confidence intervals around these trajectories are excluded for ease of interpretation; when included, there are substantial overlaps between trajectories. In general, there appears to be a degree of consistency in estimates between baseline age groups as each baseline age group ages, demonstrating that birth cohort effects are not adversely impacting on mental health scores. Simply, our prototypical 18 year old would approximate the same mental health score at 35 years of age, 17 years later, as a 35 year old reported at baseline. The trajectories demonstrate that in line with our earlier age analyses, there is an improvement in mental health as people age, with better mental health reported by those 65-74 years of age, then followed by a substantial decline in mental health among those aged $75+$.

\section{Discussion}

The purpose of this study was to examine the long-term state of Australia's mental health using a large nationally representative household sample of individuals who have provided annual mental health data over a 17-year period. It is noted that among the youngest and oldest adults, and among males more generally, there has been a small decline in mental health, particularly in very recent years. Generally, mental health improves as individuals age although there is a notable decline in mental health among very old adults, supporting the findings from other Australian longitudinal studies of ageing (Burns et al., 


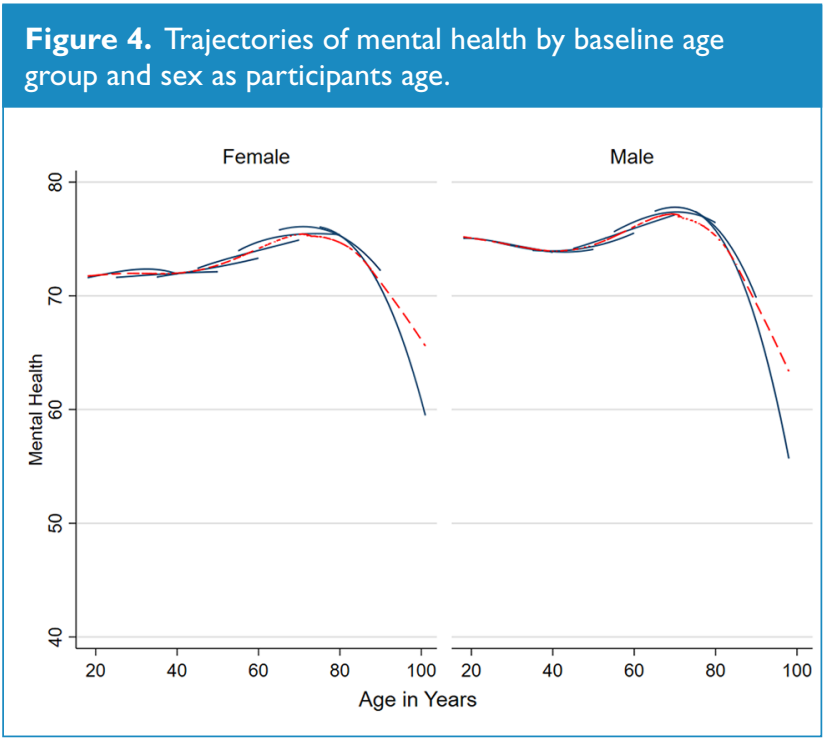

Baseline Age Group trajectories displayed in navy colour; for comparison, trajectory for those participants not at baseline displayed in red colour.

2012, 2013b). Since most of this decline appears to have occurred in recent years, continued surveillance is needed to confirm the extent to which the changes for these groups continue. However, despite these few notable changes, the overarching consistent finding has to be that there has been no substantive change in the level of mental health over the study period, for most Australians. Indeed, apart from declines of around $0.2 \mathrm{SD}$ and $0.4 \mathrm{SD}$ for the very youngest and oldest adults, the size of any change has been of negligible magnitude. And even despite these declines, most point estimates are well above that level of the mental health scale which are indicative of depression and mental ill-health more broadly. This leads us to conclude that for most Australians there has been no worsening (i.e. increasing burden) in general psychological distress. However, it also means there has been no improvement in overall population levels of mental health despite considerable public health funding expenditure and concerted public health activities that focus on drawing public attention to the burden attributed to mental ill-health.

These findings should not be surprising. Previously, Jorm (2018) has highlighted no substantive change in level of psychological distress in the Australian population using repeated cross-sectional data from the National Health Survey. From the current data, utilising annual observations from a sample of 27,519 participants over 17 years, the results confirm Jorm's findings and indeed suggest that for some groups (young, very old) a worsening of the mental health of Australians is reported. As Jorm highlighted, despite considerable public funding through the Better Access scheme, there has been no demonstrable improvement in the mental health of Australians. This may point to the need for a prevention approach (Jorm, 2014) that is comparable to the public health investment in treatment and intervention strategies.

Unlike other studies (Keyes et al., 2014), we did not find strong evidence for birth cohort effects. Our results showed concordance in mental health estimates between baseline age groups and each group as it aged. We are therefore confident regarding the robustness of our findings and that purported age differences do indeed reflect contextual, age-related or developmental changes. And given that the oldest participants reported the most substantive declines in mental health, serious concern regarding the need to address these declines is warranted. While current mental health activities are quite rightly directed towards teenagers and young adults (McGorry et al., 2007), a time where many report their first mental ill-health episode (Kessler et al., 2005), there is clearly a need for mental health advocates to question what public health initiatives are being adequately directed to the oldest-old in our community to address these impacts.

There are several limitations of this study that must be acknowledged. Most notable is that the MHI-5 is a general measure of mental health or psychological distress rather than a specific measure of clinical diagnosis. However, the SF-36 mental health scale is one of the most widely used measures of mental health in epidemiological and public health (McCabe et al., 1996; Rumpf et al., 2001; Skapinakis et al., 2005; Ware and Gandek, 1998) and has been validated in the HILDA Survey previously (Butterworth and Crosier, 2004). Also, while the study is itself not specifically a health study, health is a secondary outcome, the sample is less likely to be biased by self-selection. We also recognise that HILDA is a household-based community survey and may miss those with more severe mental health not living in the community or are not in stable household.

To conclude, the findings suggest that estimates of mental health have been relatively stable in Australia over the last 17 years and support other findings (Jorm, 2018) which point to a lack of impact from current mental health initiatives. This is particularly notable for the youngest participants who have reported some decline in recent years. But, it also emphasises a current lack of public investment in addressing the more substantive declines in the mental health of older Australians. Our findings indicate that the oldest of participants have reported the most adverse declines in mental health. Given the lack of improvement for most, the findings also emphasise that future public health initiatives may need to transcend a treatment-focus on those at high-risk, and consider more preventive approaches for those in the population at low-average risk.

\section{Acknowledgements}

This paper uses unit record data from the Household, Income and Labour Dynamics in Australia (HILDA) Survey. The HILDA Project was initiated and is funded by the Australian Government Department of Social Services (DSS) and is managed by the Melbourne Institute of Applied Economic and Social Research 
(Melbourne Institute). The findings and views reported in this paper, however, are those of the author and should not be attributed to either DSS or the Melbourne Institute.

\section{Declaration of Conflicting Interests}

The author(s) declared no potential conflicts of interest with respect to the research, authorship and/or publication of this article.

\section{Funding}

The author(s) received no financial support for the research, authorship and/or publication of this article.

\section{ORCID iDs}

Richard Andrew Burns (iD https://orcid.org/0000-0002-77504341

Dimity A Crisp iD https://orcid.org/0000-0003-0241-1121

\section{Supplemental Material}

Supplemental material for this article is available online.

\section{References}

Anstey KJ, Burns RA, Birrell CL, et al. (2010) Estimates of probable dementia prevalence from population-based surveys compared with dementia prevalence estimates based on meta-analyses. $B M C$ Neurology 10: 62.

Australian Bureau of Statistics (ABS) (2002). 4364.0 - National Health Survey: Summary of Results, 2001. Canberra, ACT, Australia: ABS.

Australian Bureau of Statistics (ABS) (2015) 4364.0.55.001 - National Health Survey: First Results, 2014-15. Canberra, ACT, Australia: ABS.

Bell A and Jones K (2015) Age, Period and Cohort Processes in Longitudinal and Life Course Analysis: A Multilevel Perspective. In: Burton-Jeangros C, Cullati S, Sacker A and Blane D (eds) A Life Course Perspective on Health Trajectories and Transitions. Life Course Research and Social Policies, Volume 4. Cham: Springer. Available at: https://link.springer.com/chapter/10.1007/978-3-319-20484-0_10

Burns RA, Birrell CL, Steel D, et al. (2013a) Alcohol and smoking consumption behaviours in older Australian adults: Prevalence, period and socio-demographic differentials in the DYNOPTA sample. Social Psychiatry and Psychiatric Epidemiology 48: 493-502.

Burns RA, Butterworth P, Luszcz M, et al. (2013b) Stability and change in level of probable depression and depressive symptoms in a sample of middle and older-aged adults. International Psychogeriatrics 25: 303-309.

Burns RA, Butterworth P, Windsor TD, et al. (2012) Deriving prevalence estimates of depressive symptoms throughout middle and old age in those living in the community. International Psychogeriatrics 24: 503-511.

Butterworth P and Crosier T (2004) The validity of the SF-36 in an Australian National Household Survey: Demonstrating the applicability of the Household Income and Labour Dynamics in Australia (HILDA) Survey to examination of health inequalities. BMC Public Health 4: 44.

Gill SC, Butterworth P, Rodgers B, et al. (2006) Mental health and the timing of men's retirement. Social Psychiatry and Psychiatric Epidemiology 41: 515-522.

Jorm AF (2014) Why hasn't the mental health of Australians improved? The need for a national prevention strategy. Australian \& New Zealand Journal of Psychiatry 48: 795-801.

Jorm AF (2018) Australia's 'better access' scheme: Has it had an impact on population mental health? Australian \& New Zealand Journal of Psychiatry 52: 1057-1062.

Kessler RC, Berglund P, Demler O, et al. (2005) Lifetime prevalence and age-of-onset distributions' of DSM-IV disorders in the national comorbidity survey replication. Archives of General Psychiatry 62: 593-602.

Keyes KM, Nicholson R, Kinley J, et al. (2014) Age, period, and cohort effects in psychological distress in the United States and Canada. American Journal of Epidemiology 179: 1216-1227.

McCabe CJ, Thomas KJ, Brazier JE, et al. (1996) Measuring the mental health status of a population: A comparison of the GHQ-12 and the SF-36 (MHI-5). British Journal of Psychiatry 169: 517-521.

McGorry PD, Purcell R, Hickie IB, et al. (2007) Investing in youth mental health is a best buy. Medical Journal of Australia 187: S5-S7.

Rumpf HJ, Meyer C, Hapke U, et al. (2001) Screening for mental health: Validity of the MHI-5 using DSM-IV Axis I psychiatric disorders as gold standard. Psychiatry Research 105: 243-253.

Skapinakis P, Lewis G, Araya R, et al. (2005) Mental health inequalities in Wales, UK: Multi-level investigation of the effect of area deprivation. British Journal of Psychiatry 186: 417-422.

Slade T, Johnston A, Oakley Browne MA, et al. (2009) 2007 National Survey of Mental Health and Wellbeing: Methods and key findings. Australian \& New Zealand Journal of Psychiatry 43: 594-605.

StataCorp (2015) Stata Statistical Software: Release 14. College Station, TX: StataCorp LP.

Vigo D, Thornicroft G and Atun R (2016) Estimating the true global burden of mental illness. The Lancet Psychiatry 3: 171-178.

Ware JE Jr and Gandek B (1998) Overview of the SF-36 Health Survey and the International Quality of Life Assessment (IQOLA) Project. Journal of Clinical Epidemiology 51: 903-912.

Ware JE Jr and Sherbourne CD (1992) The MOS 36-Item Short-Form Health Survey (SF-36). I. Conceptual framework and item selection. Medical Care 30: 473-483.

Wasserstein RL, Schirm AL and Lazar NA (2019) Moving to a world beyond ' $\mathrm{p}<0.05$ '. The American Statistician 73: 1-19.

Whiteford HA, Degenhardt L, Rehm J, et al. (2013) Global burden of disease attributable to mental and substance use disorders: Findings from the Global Burden of Disease Study 2010. The Lancet 382: $1575-1586$.

Wooden M and Watson N (2007) The HILDA survey and its contribution to economic and social research (so far). Economic Record 83: 208-231.

Yamazaki S, Fukuhara S and Green J (2005) Usefulness of five-item and three-item Mental Health Inventories to screen for depressive symptoms in the general population of Japan. Health and Quality of Life Outcomes 3: 48. 